\title{
Enseignement de l'anglais de spécialité en génie électrique et informatique industrielle
}

Documents authentiques, niveaux de compétence professionnelle et niveaux de langue

\section{Monique Mémet}

\section{(2) OpenEdition}

\section{Journals}

Édition électronique

URL : http://journals.openedition.org/asp/4410

DOI : 10.4000/asp.4410

ISSN : 2108-6354

\section{Éditeur}

Groupe d'étude et de recherche en anglais de spécialité

Édition imprimée

Date de publication : 1 mars 1993

Pagination : 575-591

ISSN : 1246-8185

\section{Référence électronique}

Monique Mémet, «Enseignement de l'anglais de spécialité en génie électrique et informatique industrielle», ASp [En ligne], 1 | 1993, mis en ligne le 05 juin 2014, consulté le 19 avril 2019. URL

http://journals.openedition.org/asp/4410; DOI : 10.4000/asp.4410

Ce document a été généré automatiquement le 19 avril 2019

Tous droits réservés 


\section{Enseignement de l'anglais de spécialité en génie électrique et informatique industrielle}

Documents authentiques, niveaux de compétence professionnelle et niveaux de langue

\section{Monique Mémet}

1 À l'heure où les fabricants de matériel informatique préparent des systèmes conviviaux où les claviers seront remplacés par des systèmes de reconnaissance vocale ou de reconnaissance de l'écriture, et par des systèmes de simulation (Caudill 1992), il nous semble essentiel de participer, de façon très modeste, à ces interactions entre l'homme et la machine.

2 Les enseignants de langues de spécialité, et notamment d'anglais de spécialité du domaine scientifique et technique, ont la possibilité de jouer un rôle d'interface pour les apprenants. Il n'est naturellement pas question de leur demander de se transformer en enseignants d'électronique, d'automatique, d'informatique ou d'une des nombreuses disciplines pour lesquelles les compétences en langue anglaise sont devenues indispensables. Les interfaces sont d'ordre relationnel (entre les enseignants des diverses disciplines), pratique (organisation des données, des supports à leur disposition), et bien sûr, linguistique.

3 Documents authentiques, génie électrique et informatique industrielle, niveaux de compétence professionnelle, niveaux de langue font partie des concepts que nous nous proposons d'organiser, de relier à l'aide d'outils informatiques.

4 L'objectif est d'aider à la découverte et à l'organisation de ces outils au sein du lieu d'établissement et aussi de faciliter une diffusion vers l'extérieur. La situation de l'enseignant de spécialité offre des similitudes avec celle des petites et moyennes entreprises ou industries (PME/PMI) : celles-ci sont en effet équipées en microordinateurs, mais ont un besoin impératif d'organiser ce matériel en réseau à l'intérieur 
de l'entreprise et ensuite vers l'extérieur, en particulier pour pouvoir affronter le nouveau marché européen de 1993.

\section{Documents authentiques}

5 Reprenons le premier terme «Documents authentiques ». Le thème n'est pas nouveau: un premier séminaire a eu lieu sur le sujet à Nancy en 1978, «L'utilisation de documents authentiques dans l'enseignement. Apprentissage des langues de spécialité » (CRAPEL 1978). Les auteurs anglo-saxons le présentent dans de nombreux ouvrages de référence (Robinson 1980 : 5-14 ; Swales 1985 ; Hutchinson \& Waters 1986 : 106).

Nous rappelons la définition donnée par Louis Trimble :

Genuine materials are those taken directly from a printed source and presented

without alteration. (1985: 27)

Notre propos est de remonter en amont, en quelque sorte de rappeler les sources dans lesquelles pourraient puiser les enseignants en langues de spécialité. Les documents, les outils de didactisation, existent, mais ils ne sont pas toujours connus des utilisateurs potentiels, en l'occurrence de l'enseignant qui prend en charge un cours d'anglais de spécialité dans ce domaine.

8 L'apprentissage linguistique s'effectue à l'aide de supports écrits (les textes), sonores et/ ou visuels (bandes audio et vidéo), de logiciels, etc., formes qui, d'ailleurs, seront regroupées, dans un avenir plus ou moins lointain selon les lieux d'enseignement, en documents multimédias. L'objet de notre étude porte sur le premier groupe de matériau, le support écrit. Le point abordé est celui de la typologie et de la connaissance du terrain et des documents. Le domaine est celui des apprenants concernés, en l'occurrence des étudiants en Institut Universitaire de Technologie, dans un Département de Génie électrique et informatique industrielle.

\section{Génie électrique et informatique industrielle}

L'origine étymologique du substantif "génie» (qui est encore la source de nombre de jeux de mots de la part des étudiants) permet de retracer en quelques lignes, le chemin technologique parcouru au cours de plusieurs siècles. Le terme, apparu en 1532, est dérivé du latin " genius » et l'acception qui nous intéresse date de la création, en 1759, du corps de troupes, le "génie militaire", "arme, service essentiellement techniques chargés de travaux de tous ordres, notamment de la construction et de l'entretien des casernements, de la fortification permanente $»^{1}$. Ce corps sera ensuite suivi par la création du génie maritime, corps d'ingénieurs militaires chargés des constructions navales et de corps civils, « le génie civil ». Le glissement correspond ainsi à l'évolution de l'organisation de la société. Le concept "génie électrique » est lié aux développements des connaissances en électricité au $19^{\mathrm{e}}$ siècle (expériences de Faraday, Gramme entre autres). La première application pratique de l'électricité date de 1837 : le télégraphe inventé par Samuel F. B. Morse) $)^{2}$. Le terme anglo-saxon "engineering", dérivé du vieux français «ingénieur ", paraît moins surprenant aux novices (mais, le terme "engineer » perd de nos jours sa charge sémantique aux États-Unis : par exemple, le mot composé de " garbage man » est remplacé par « sanitary engineer »). 
10 L'analyse linguistique des documents authentiques impose la compréhension du milieu dans lequel doivent naviguer les apprenants, ainsi que la connaissance de leur niveau de culture scientifique. Pour appréhender ces derniers, des interactions entre linguistes et scientifiques sont fort utiles, voire indispensables. L'interdisciplinarité a été évoquée depuis la création même de ces établissements (en 1966³) par Evelyne Brouzeng (1982) et Anne Pradeilles en particulier (1982). Les enseignants linguistes sont amenés à acquérir non pas les connaissances professionnelles de leurs apprenants, mais la connaissance du code culturel de la spécialité, une vision diachronique et synchronique des disciplines scientifiques étudiées. La compréhension de la terminologie requiert la maitrise des concepts de base, ainsi qu'une culture scientifique de base, le noyau (" the common core »), et des rudiments de la spécialité. Des chercheurs scientifiques comme Gilles de Gennes, prix Nobel 1991, ou Hubert Reeves, astrophysicien, permettent aux néophytes, grâce à leurs travaux de vulgarisation et à leurs réflexions (cf. Reeves 1990), de saisir la complexité du savoir scientifique. La vision globale de ces sciences, et la connaissance du développement de la technologie sont tout aussi nécessaires au linguiste d'anglais de spécialité scientifique que la vision de l'économie mondiale (globale) l'est au dirigeant d'une petite entreprise française, à l'aube du $21^{\mathrm{e}}$ siècle.

11 Cette approche est une démarche de formation continue personnelle. La collecte d'informations est en elle-même une démarche heuristique : l'enseignant est confronté aux difficultés de compréhension d'un savoir scientifique, tout comme l'apprenant est confronté aux difficultés du savoir linguistique.

12 Nous nous proposons de définir une stratégie pour la collecte de matériaux, supports didactiques, de suggérer les méthodes de classification qui tiennent compte des compétences professionnelles, en rappelant les travaux effectués dans ce domaine. Nous présentons une organisation structurée de ces données à l'aide d'un logiciel (FileMaker $\mathrm{Pro}^{\circledR}$ ) qui permet d'alimenter une banque de données pour le Département de Langues Vivantes Pratiques de Bordeaux 2. L'organisation des données est un outil de départ pour une étude lexicale, discursive et syntaxique de l'anglais de spécialité dans le domaine du Génie électrique et de l'informatique industrielle.

13 À noter que les termes "Génie électrique » et « informatique industrielle » ne désignent pas exclusivement des sciences ou des disciplines d'enseignement, mais également 44 départements d'Instituts Universitaires de Technologie en France qui regroupaient 10500 étudiants, à la rentrée universitaire 1991.

\section{Compétences langagières et compétences professionnelles}

14 La cible de l'enseignement est un public hétérogène, par ses compétences langagières en langue $2^{4}$, et par ses savoir et savoir-faire dans les disciplines scientifiques et techniques. L'hétérogénéité provient de la composition même des groupes, c'est-à-dire de l'origine scolaire des apprenants (sections techniques : F2, F3, ou scientifiques C, D ou E). À ce stade d'apprentissage, il est prématuré de parler de compétences professionnelles; on observe des différences de savoir technique entre les étudiants qui ont acquis des connaissances au cours de leur apprentissage scolaire (dans les sections techniques des lycées), ainsi que dans leurs activités de loisirs. La maîtrise de l'outil informatique («computer literacy») que possèdent quelques étudiants a une influence directe sur le comportement à l'égard 
de supports didactiques écrits utilisés pendant les cours d'anglais. En effet, pour certains, une grande partie de la documentation qu'ils ont utilisée pour leurs systèmes personnels est en langue anglaise. En revanche, pour certains étudiants, même la configuration d'un ordinateur est encore une découverte en début d'année.

Nous avons fait référence à des documents utilisés pendant les cours de langues. Quels sont ces documents? Sont-ils imposés par des programmes? Les programmes des enseignements en IUT sont définis par les Commissions pédagogiques nationales (CPN), constituées par l'Assemblée des Chefs de département ${ }^{5}$. Les programmes, nationaux, couvrent tous les enseignements dispensés dans les établissements, y compris l'anglais. D’après les derniers textes parus, rédigés en 1985 et révisés en 1987, la nature des documents est indiquée dans le programme: «recueils de caractéristiques, notices d'emploi, articles scientifiques et techniques, etc.» (Brochure Éducation nationale 1985 : 73-75). Il est suggéré l'étude d'un lexique, mais la nature de ce dernier n'est qu'indicative : « des documents pour spécialistes » (op. cit. : 75).

\section{Documents pour spécialistes}

Quels sont ces "spécialistes»? Deux catégories de spécialistes convergent: les enseignants des disciplines scientifiques et techniques, et les utilisateurs dans l'industrie, ingénieurs et techniciens supérieurs. Quels sont les critères à prendre en compte pour délimiter les « documents pour spécialistes »?

17 La dualité déterminée par Pauline Robinson (1980 : chapitre 1) «English for Occupational Purposes » (EOP) et «English for Educational Purposes » (EEP) ne correspond pas exactement à la situation de l'enseignement en Génie électrique et informatique industrielle (GE\&II).

Un premier groupe de documents comprend ceux utilisés par les enseignants des disciplines scientifiques et techniques comme supports de cours, les documents distribués aux étudiants (polycopiés) ou la documentation à la disposition des étudiants lors de leurs séances de travaux dirigés (intitulés "travaux de réalisation", "maquettes", etc.). La deuxième catégorie comprend les documents utilisés dans l'industrie, au sein des entreprises, dans des emplois similaires à ceux que les futurs titulaires de DUT occuperont. Il y a une autre catégorie à ajouter aux deux précédentes, celle constituée par les documents que les enseignants utilisent à des fins de recherche ou d'intérêt personnel concernant les domaines en question.

Où trouver ces documents ? Pour la première catégorie, la collecte est aisée : soit auprès des enseignants, soit auprès des techniciens qui font partie de l'encadrement de tout département GE\&II. La lecture, ou plutôt le survol, par le linguiste des polycopiés des cours scientifiques et techniques offre un double intérêt : l'appréciation de la quantité de documentation en langue anglaise (parfois les deux tiers du polycopié), et le contenu scientifique des documents. L'enseignant d'anglais de spécialité ne va pas devenir, bien sûr, un scientifique qui aurait une maîtrise totale des concepts relatifs aux diverses disciplines enseignées aux apprenants auxquels il va s'adresser. Même s'il le désirait, cela serait irréalisable. En revanche, la compréhension des principes de base de ces diverses disciplines est indispensable et elle n'est pas impossible. Un ingénieur américain, Henry Petroski, dans son dernier livre, The Pencil, qui pétille d'humour le formule ainsi :

I believe that anyone today is capable of comprehending the essence of, if not of contributing to, even the latest high technology. Behind all the jargon, 
mathematics, science, and professionalism of engineering lies a method as accessible and pervasive as the air we breathe. (1990 : ix-x)

20 Un deuxième moyen de connaître le terrain et aussi de collecter des documents authentiques consiste à contacter les entreprises. Aimée Blois avait abordé la question en faisant une enquête auprès des entreprises pour rendre compte de la pratique de l'anglais dans l'industrie. Les résultats sont publiés dans les Cahiers de l'APLIUT (Blois 1985). De tels contacts sont automatiques dans le cadre de la formation continue. Voir à ce sujet le numéro des Langues modernes consacré à la formation des adultes, et en particulier les articles de Michel Perrin (1992) et de Claude Springer. Ce dernier traite de l'identification des «besoins langagiers des organisations professionnelles» (1992). Son approche, dans le cadre de la formation continue (GRETA), permet de cibler des compétences professionnelles de façon bien plus précise qu'il n'est possible de le faire pour des formations initiales en IUT, ou autres formations similaires. En effet, la formation donnée dans ces établissements est pluridisciplinaire ; elle vise à permettre aux titulaires d'un DUT d'être opérationnels dans de nombreux secteurs d'activité.

21 Recueillir des documents auprès d'entreprises très diverses n'est pas aisé. Pour citer un exemple personnel, un courrier envoyé en 1990 et destiné à recueillir des documents pour un enseignement à des étudiants en IUT, dans un département secondaire, Organisation et Gestion de la Production a suscité un taux de réponse de $29 \%$, légèrement supérieur au taux de réponse habituel. Toutefois, les documents authentiques transmis par les entreprises présentaient souvent peu d'intérêt. Les entreprises ont fait parvenir leur plaquette de présentation ou leur bilan d'exercice traduits en anglais. La raison de l'envoi de tels documents peut s'expliquer de diverses façons :

- le courrier a été remis à la personne chargée de la communication dans l'entreprise ; cette dernière a voulu diffuser l'image de marque de son entreprise, sans tenir compte de la demande pourtant formulée avec précision ;

- les ingénieurs concernés n'ont souvent ni le temps, ni le désir, de diffuser des documents d'usage courant, qui peuvent contenir des informations à ne pas divulguer...

À moins de se rendre soi-même dans les entreprises et de cibler uniquement les services concernés, de tels documents ne peuvent être exploitables à des fins didactiques.

Nous avons mentionné une troisième catégorie de documents, ceux utilisés par les enseignants à la fois dans le cadre de leur enseignement, ainsi que dans celui de leur recherche universitaire et personnelle. Cette catégorie de documents permet au linguiste de mettre en relation le degré de spécialisation du document authentique avec le niveau d'énonciation du discours. Sans cette mise en perspective, l'enseignant de spécialité ne peut envisager la culture professionnelle dans son ensemble. Les enseignants du département GE\&II d'Évry ont répondu à un questionnaire concernant les ouvrages en langue anglaise qu'ils utilisaient. Le nombre d'exemplaires cité était en grande partie lié à la discipline enseignée et au statut de chercheur.

Une dernière catégorie de documents authentiques peut être recueillie dans les pays de langue anglaise : ce sont des documents utilisés par les étudiants, débutants ou confirmés, documents d'auto-apprentissage des techniques avec divers degrés de didacticité et de niveau de spécialisation lexicale. 


\section{Typologie des documents authentiques}

La mise en pratique de ces quatre stratégies (recueillir des documents utilisés dans les cours par les enseignants; recueillir des documents utilisés par les enseignants euxmêmes; collecter des informations auprès des entreprises et rechercher les manuels anglo-saxons de la spécialité) a permis d'esquisser une typologie des documents authentiques dans le domaine du Génie électrique et de l'informatique industrielle.

Une telle typologie a été établie par Christiane Beaufrère-Bertheux dans le domaine de l'anglais de la chimie (1983). Elle regroupe les documents du discours de la chimie en neuf catégories: "publications officielles d'information scientifique; publicités pour appareillage ; revues scientifiques de bon niveau, non spécialisées ; catalogues de produits et appareils ; ouvrages didactiques; nomenclature (ex. : normes, iconographies, etc.) ; notices de montage, d'utilisation et d'entretien ; brevets ; publications spécialisées ».

Dans un article récent, Gregory A. Myers (1992) analyse un segment de cette production, les manuels scientifiques et techniques universitaires, sous l'angle de l'incidence de ces manuels dans la vie d'un scientifique d'une part, et dans « la vie d'un fait », d'autre part. Il cite le classement établi par J. Ziman concernant la reconnaissance d'un fait scientifique :

[Ziman] places textbooks near the end of the process, from journal articles and articles citing them and review articles and finally to textbooks, encyclopedia articles, and undergraduate lectures. (1992:7)

\section{Critères d'organisation des documents authentiques}

28 Pour établir une typologie des documents authentiques, les éléments suivants peuvent être retenus : le destinataire potentiel du document, le degré de compétence scientifique requis pour la compréhension du message véhiculé, le site d'utilisation du document écrit, l'énonciateur.

9 Le destinataire potentiel peut être: le praticien de l'industrie (ingénieur, technicien supérieur) ; l'enseignant chercheur (laboratoire de recherche, université, Grande École); l'enseignant d'un établissement secondaire ; l'étudiant (licence, maîtrise, DEA, doctorat) ; l'amateur (hobbyist, do-it-selfer).

30 Le degré de compétence scientifique requis pour la compréhension du message véhiculé, la compétence professionnelle, couvre un large spectre : du chercheur spécialisé (public restreint) au degré de vulgarisation le plus grand.

Le site d'utilisation: le bureau d'études; l'entreprise; l'usine (services de production, réalisation) ; les établissements d'enseignement ; le laboratoire de recherche.

32 L'énonciateur : le chercheur de haut niveau (type CNRS, ingénieur de développement); le chercheur de catégorie moyenne (enseignant chercheur); l'auteur de manuels; le vulgarisateur (journaliste spécialisé, généraliste, etc.). Il va de soi que les catégories ont de multiples corrélations.

3 Nous avons dressé un inventaire, non exhaustif, des documents pour «spécialistes » et nous donnons pour chaque catégorie quelques exemples.

\section{Publications spécialisées}


De nombreuses banques de données fournissent des abstracts (par exemple CEDOCAR en France).

2. Brevets

3. Notices

tices de montage

Unpacking and installing your DN/3000/DN4000 Personal Workstation and the DSP3000/DSP4000 Server. Apollo Computer Inc., 1987.

3.2. Notices d'utilisation

VAX Systems Hardware Handbook - VAXBI Systems, Digital, 1988.

40 3.3. Notices d'entretien

4. Catalogues produits et systèmes (" Data books »)

LS/S/TL Logic Data book. Santa Clara : National Semiconductor, 1989.

5. Revues techniques spécialisées

41 5.1. Systèmes de traitement de l'information

Byte

PC World

Electronic Design

Electronics

European Electrooptics

44 5.4. Automatique

Automatica

Robotica

6. Ouvrages didactiques, manuels

6.1. Handbooks

Jones, Thomas H. Electronic Components Handbook. Reston : Prentice-Hall Company, 1978. Ryan, Jeremy. Electronic Assembly. Reston : Prentice-Hall Company, 1980.

Collection Made Simple ${ }^{\circledast}$ Books: Freeman, Ira M. Physics Made Simple, Revised Edition. 
NewYork : Doubleday, 1990.

Collection The Essentials ${ }^{\circledR}$ : The Essentials of Electronics I. Piscataway, REA, 1990.

« Do-it-yourself books »

Collins, Frederick A. revised by Robert Hertzberg. The Radio Amateur's Handbook, 15th revised ed. New York : Harper and Row, Publishers, 1983.

\section{Encyclopédies}

Encyclopoedia Britannica, Macropedia, 1991.

Collier's Encyclopedia, New York : Macmillan Education Company, 1989.

\section{Dictionnaires spécialisés}

Sinclair, Ian R. Dictionary of Electronics. Glasgow : Collins, 1988.

\section{Revues de vulgarisation}

49 9.1. Scientifiques non spécialisées

New Scientist

Science News

9.2. Grand public (sections « science et technologie »)

Newsweek

Time

9.3. Monde des affaires (sections « science et technologie »)

Business Week

The Economist

10. Publicités pour produits, systèmes et services

10.1. Prospectus, plaquettes, etc. fournis par l'entreprise

Genesis, ICONIX, USA.

\subsection{Publicités, annonces dans la presse écrite}

Certains magazines, envoyés gratuitement aux professionnels, tels que Control Engineering ou Electronic Design, comprennent essentiellement des articles de publicité rédactionnelle.

\section{Organisation structurée des documents authentiques}

Pour que le recueil de ces informations puisse avoir une utilisation directe, nous avons entrepris de les organiser avec un logiciel.

\section{Base de données}

La création ou l'utilisation de bases de données informatiques nécessitait, il y a une dizaine d'années, la connaissance de langages de programmation (ex. : Dbase). Ce n'est plus nécessaire de nos jours, grâce à la diffusion de systèmes conviviaux (" user-friendly »). Les champs, qui contiennent les informations, sont créés en utilisant le menu déroulant du logiciel (FileMaker Pro ${ }^{\circledast}$ de Claris, pour Apple). L'intérêt d'un tel logiciel réside dans la possibilité à l'accès des informations actualisées, et une consultation pratique et rapide : l'interrogation peut se faire à partir d'un titre, d'un genre de documents, du public visé, etc., c'est-à-dire, par rapport aux rubriques qui correspondent aux champs créés.

Certaines données sont actualisées en permanence, ce sont des denrées périssables. Un système dynamique d'organisation permet aux enseignants d'anglais de spécialité de trouver rapidement les informations recherchées. Il ne s'agit pas d'une banque de données comparable aux banques de données scientifiques qui comprennent 
d'innombrables documents et sont maintenant accessibles dans la plupart des laboratoires de recherche par réseaux (Ethernet). éminemment périssables. La technologie concernée évolue avec une rapidité maintenant connue du grand public: le premier système informatisé, l'ENIAC, outil volumineux et complexe, présenté au public en 1946, n'avait pas les capacités d'une petite calculette de nos jours (Augarten 1984 : chapitre 4).

La réunion ayant pour intitulé " atelier », nous nous proposons de terminer l'exposé par une démonstration, sur ordinateur, de notre outil au début de sa phase de création.

\section{BIBLIOGRAPHIE}

Augarten, Stan. 1984. Bit by Bit. An Illustrated History of Computers. Londres : George Allen \& Unwin Publishers.

Beaufrère-Bertheux, Christiane. 1983. «L'anglais de la chimie. Une langue de spécialité parmi d'autres ». Les Cahiers de l'APLIUT 10, 51-56

Blois, Aimée. 1985. « Pédagogie de l'anglais dans un département de Génie mécanique : quelles priorités ? Présentation des résultats d'une enquête sur la pratique de l'anglais dans l'industrie ». Les Cahiers de l'APLIUT 16, 34-48.

Brochure Ministère de l'Éducation nationale. 1985. Direction des Enseignements Supérieurs. Instituts Universitaires de Technologie. Génie Électrique et Informatique Industrielle. 
Brouzeng, Evelyne. 1982. «Interdisciplinarité et langues de spécialité ». Actes des $4^{e}$ et $5^{e}$ Colloques du GERAS, 1981-1982. Bordeaux : Université Bordeaux 2, 81-87.

Caudill, Maureen.1992. « Kinder, gentler computing ». Byte, April 1992, 135-150.

CRAPEL (dir.). 1978. « L'utilisation des documents authentiques dans l'enseignement/ apprentissage des langues de spécialité. Actes du Séminaire CRAPEL-AILA, 2-4 mars 1978. Nancy : Université Nancy 2 (non relié).

Dechet, Arlette. 1990. «Stratégie pour l'enseignement de l'anglais de l'informatique : démarche didactique intégrant l'ordinateur et le traitement de texte ». Thèse de doctorat nouveau régime, Montpellier 3.

Hutchinson, Tom \& Alan Waters. 1986. English for Specific Purposes, A Learning Centred Approach. Cambridge : Cambridge University Press.

Mémet, Monique. 1992. « Fichier Périodiques Langues de Spécialité ». Les Cahiers de l'APLIUT, supplément au n44 (11/3).

Myers, Greg. 1992. «Textbooks and the sociology of scientific knowledge ». English for Specific Purposes 11/ 1, 3-17.

Perrin, Michel. 1992. «Centres de ressources et autonomie guidée ». Les Langues Modernes 1, 21-36. Petroski, Henry. 1990. The Pencil. A History of Design and Circumstance. New York : Alfred A. Knopf. Pradeilles, Anne. «L'interdisciplinarité, une formation continue indispensable à l'enseignant de langue de spécialité ». Actes des $4^{e}$ et $5^{e}$ Colloques du GERAS, 1981-1982. Bordeaux : Université Bordeaux 2, 101-113.

Reeves, Hubert. 1990. Malicorne, Réflexions d'un observateur de la nature. Paris : Seuil.

Robinson, Pauline C. 1980. ESP (The Present Position). Oxford : Pergamon Press.

Springer, Claude. 1992. « Langues et entreprises : la question de l'identification des besoins langagiers des organisations professionnelles ». Les Langues modernes 1, 37-44.

Swales, John M. 1985. Episodes in ESP. Hemel Hempstead, UK : Prentice-Hall International.

Trimble, Louis. 1985. English Science and Technology. A Discourse Approach. Cambridge : Cambridge University Press.

\section{NOTES}

1. Robert, Paul. 1966. Dictionnaire alphabétique et analogique de la langue française, Tome troisième Paris : Société du Nouveau Lettré, Le Robert, 255-257.

2. The New Encyclopcedia Britannica, Volume 18, Macropædia, 15th edition. 1991. Chicago: Encyclopædia Britannica, Inc., 414-417.

3. Arrêté du 19 novembre 1965.

4. Voir à ce sujet les travaux d'Arlette Dechet (1990). Elle présente le public d'un département informatique et recense les matériaux linguistiques à didactiser (pages 12-14).

5. Arrêté du 22 juin 1967.

6. $\mathrm{IEEE}=$ Institute of Electrical and Electronics Engineers, créé en 1963, de la fusion de American Institute of Electrical Engineers, fondé en 1884 et de the Institute of Radio Engineers, fondé en 1912.

7. Programme en service au Département DLVP de Bordeaux 2. 


\section{AUTEUR}

\section{MONIQUE MÉMET}

IUT Université Paris 12.m.memet@orange.fr 